\title{
Desmoid Tumor of the Rectus Abdominis Muscle in a Postpartum Patient
}

Seung Ho Choi, Jung Ho Lee, Bommie F. Seo, Sang Wha Kim, Jong Won Rhie, Sang Tae Ahn

Department of Plastic and Reconstructive Surgery, The Catholic University of Korea College of Medicine, Seoul, Korea

Correspondence: Jung Ho Lee

Department of Plastic and Reconstructive Surgery, The Catholic University of Korea College of Medicine, 222 Banpo-daero, Seocho-gu, Seoul 137-040,

Korea
Tel: +82-2-2258-6144, Fax: +82-2-594-7230

E-mail: tfm0822@catholic.ac.kr

No potential conflict of interest relevant to this article was reported.

Received: 4 May 2012 • Revised: 12 Jun 2012 • Accepted: 13 Jun 2012 pISSN: 2234-6163 • elSSN: 2234-6171

http://dx.doi.org/10.5999/aps.2012.39.4.439 • Arch Plast Surg 2012;39:439-441

Copyright @ 2012 The Korean Society of Plastic and Reconstructive Surgeons

This is an Open Access article distributed under the terms of the Creative Commons

Attribution Non-Commercial License (http://creativecommons.org/licenses/by-nc/3.0/) which permits unrestricted non-commercial use, distribution, and reproduction in any medium, provided the original work is properly cited.

Desmoid tumors, also known as aggressive fibromatosis, are uncommon mesenchymal neoplasms. They account for only $0.03 \%$ of all tumors and $3 \%$ of all soft-tissue neoplasms [1]. A pregnancy-associated desmoid tumor is an even less common disease very rarely reported in published articles. Although it grows by locally infiltrating its neighboring tissues and structures, it is devoid of metastatic evolution. It usually occurs between the ages of 25 and 40 years, with a strong prevalence among women in the fertile age group [1].

A 36-year-old woman presented with a painless mass in the right upper quadrant of her abdomen. She had discovered the mass six months earlier and stated that the size of the mass had not increased. She had no relevant family history of related ailments such as of the familial adenomatous polyposis syndrome 
or the Gardner syndrome; did not smoke and drink alcohol; and was not taking any medication. She had given birth to two children by normal spontaneous delivery, the last of which was 3 years earlier. She had no history of trauma or surgery. Her laboratory findings showed no abnormality. A physical examination showed a $3 \times 3 \mathrm{~cm}$ firm mass that palpated and was fixed to the patient's abdominal wall. A computed tomography (CT) scan revealed a hypodense mass in the right rectus muscle and mild enhancement in the delayed images after intravenous administration of a contrast medium (Fig. 1). It was completely removed with some normal muscle tissue around it under general anesthesia. A round mass with a firm, gritty texture was located in the right rectus muscle, and it had unclear boundaries (Figs. 2, 3). A histopathologic study confirmed the diagnosis of a desmoid tumor with a clear surgical margin. It revealed the typical cell pattern of myofibroblasts and fibroblasts. There were uniform, plump, and immature spindle cells that had bland, indistinct nuclei (Fig. 4). The postoperative course was uneventful, and the patient was discharged on the first postoperative day. She was still well at her 6 month follow-up, with no evidence of tumor recurrence.

Desmoid tumors can be classified into three groups: intra-abdominal, within the abdominal wall,

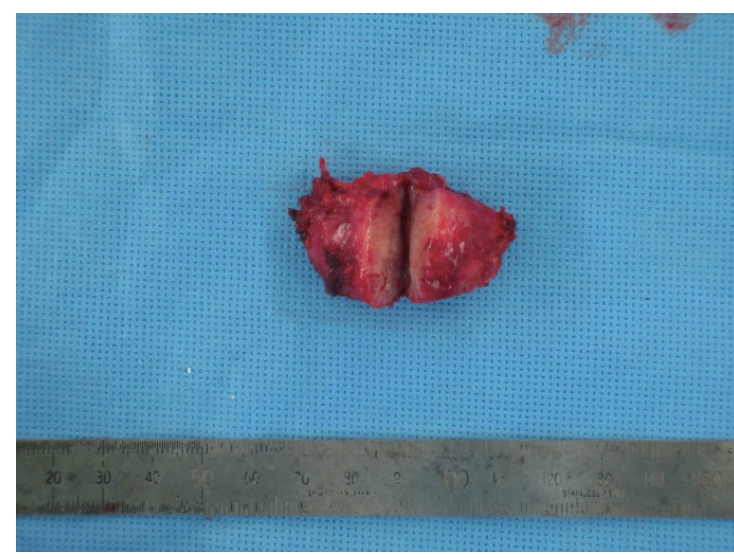

Fig. 3 . The $3 \times 3 \times 2 \mathrm{~cm}$ round mass with some normal peripheral muscle tissue (split into two; after removal).

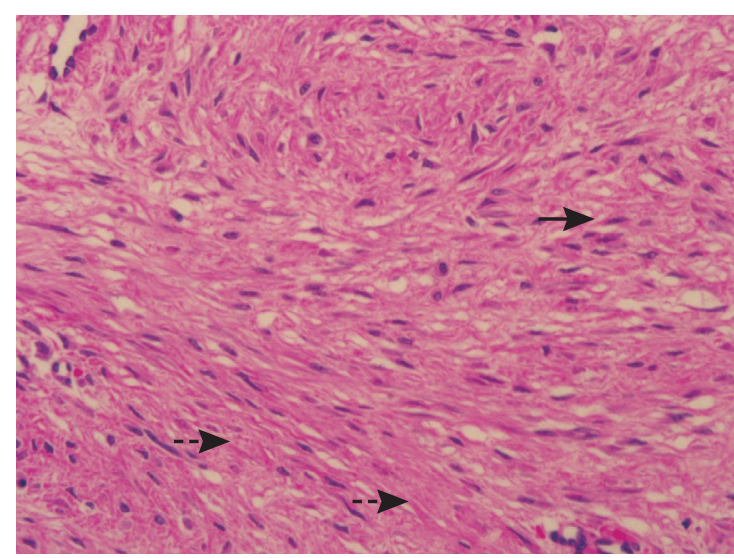

Fig. 4 .

Histopathologic examination finding of the typical elongated cell pattern of myofibroblasts and fibroblasts (black arrows) with abundant collagen (black dashed arrows). Uniform, plump, and immature spindle cells with bland, indistinct nuclei are seen $\left(H \& E_{1} \times 400\right)$. 
and extra-abdominal. The histologic findings on these lesions are identical. Sixty percent of desmoids are extra-abdominally located, and $15 \%$ appear in the abdominal cavity. Another $25 \%$ of desmoids are in the abdominal wall [2]. Extra-abdominal desmoid tumors are widely distributed but are commonly found in the shoulder girdle, trunk, and lower extremities. Most intra-abdominal desmoid tumors occur in patients with familial adenomatosis polyposis, and when these two conditions occur together, they are often called "Gardner syndrome."

A clear link between the development of abdominal wall desmoid tumors and the pregnancy and postpartum state has appeared in many case reports in the literature [3]. Interestingly, in most of these cases, desmoid tumors almost always occur in the abdominal wall of postpartum women, and there is a predilection for the right rectus abdominis muscle, as in this case.

The definite etiology of desmoid tumors, whether pregnancy-associated or otherwise, is currently unknown. It has been postulated that the hormonal and immunologic changes in the pregnant or postpartum state appear to be important factors. The local effect due to the mechanical stress from a gravid uterus may also contribute to the occurrence of a desmoid tumor [4].

Histologically, desmoid tumors consist of elongated fibroblasts and myofibroblasts characterized by elongated, tapered cytoplasm; elongated, vesicular, and typical appearing nuclei; and multiple small nucleoli. The cells are linearly arranged and surrounded and separated from each other by collagen [1].

Ultrasonography has shown that desmoid tumors appear as well-defined lesions with variable echogenicity. Their lateral borders may occasionally appear ill-defined or irregular [1]. The CT appearance of desmoid tumors depends on their composition. They may appear homogeneous or heterogeneous and hypo-, iso-, or hyperintense compared with the attenuation of the muscles. The degree of their enhancement after the intravenous administration of a contrast medium is variable. The magnetic resonance imaging (MRI) features of desmoid tumors also show wide variability depending on the stage in which they are imaged. Characteristic MRI findings include poor margination, low signal intensity on T1-weighted images and heterogeneity on T2-weighted images, and variable contrast enhancement. MRI may have a prognostic value because a higher $\mathrm{T} 2$ signal is reportedly associated with faster growth [5].

The treatment options for desmoid tumors can vary according to the individual patient's condition
[3]. Stable asymptomatic desmoids can be observed and followed up with imaging at a 3- to 6-month interval. Treatment is necessary for symptomatic patients, especially those with a mass effect on critical structures. Surgery is the mainstay treatment in patients with extra-abdominal desmoid tumors, including abdominal wall desmoid tumors, as in this case. Though a few authors report that recurrence is independent of the negative margin, most investigators recommend resection with a wide margin of surrounding normal tissue to minimize the risk of local recurrence. In addition, a retrospective analysis of 189 desmoid tumor patients revealed that the presence of a tumor at the microscopic surgical margin was the single most significant determinant of the recurrence of desmoid tumor in patients treated with surgery. Thus, in some cases of large desmoid tumors, reconstruction procedures such as transposition of aponeurotic-muscular flaps or use of a mesh product should be considered preoperatively [4].

In conclusion, the diagnosis of desmoid tumor should be strongly considered in female patients who have experienced childbirth presenting with an abdominal wall mass even though it is a rare entity. Our case of desmoid tumor in a postpartum patient, a very rare occurrence, was completely treated with surgery and is under follow-up without any relapse.

\section{References}

1. Economou A, Pitta X, Andreadis E, et al. Desmoid tumor of the abdominal wall: a case report. J Med Case Rep 2011;5:326.

2. De Cian F, Delay E, Rudigoz RC, et al. Desmoid tumor arising in a cesarean section scar during pregnancy: monitoring and management. Gynecol Oncol 1999;75: $145-8$.

3. Carneiro C, Hurtubis C, Singh M, et al. Desmoid tumors of the right rectus abdominus muscle in postpartum women. Arch Gynecol Obstet 2009;279:869-73.

4. Johner A, Tiwari P, Zetler P, et al. Abdominal wall desmoid tumors associated with pregnancy: current concepts. Expert Rev Anticancer Ther 2009;9:1675-82.

5. Shinagare AB, Ramaiya NH, Jagannathan JP, et al. A to $\mathrm{Z}$ of desmoid tumors. AJR Am J Roentgenol 2011;197:W1008-14. 